\title{
Should women at high risk of breast cancer be screened with MRI?
}

Leach MO, Boggis CR, Dixon AK, Easton DF, Eeles RA, Evans DG, et al; MARIBS study group. Screening with magnetic resonance imaging and mammography of a UK population at high familial risk of breast cancer: a prospective multicentre cohort study (MARIBS). Lancet 2005;365(9473):1769-78.

Background: Women at a high risk of breast cancer often develop the disease at an early age. In young women, mammography has poor sensitivity because their breast tissue is denser. Use of contrast-enhanced (CE) MRI may improve the detection of cancer in this high-risk group.

Design: Women with a family history or a high probability of having a $B R C A 1, B R C A 2$ or TP53 mutation were enrolled from 22 centres in the United Kingdom and offered annual screening with both CE MRI and mammography. The cohort was prospectively followed for 2-7 years.

Results: The authors found that CE MRI had a higher sensitivity than mammography, but a lower specificity, consistent with two 2004 reports $^{1,2}$ (Table 1). Most cancers were detected in women with $B R C A 1$ or who had a relative with that mutation; in this subgroup, the sensitivity of CE MRI was $92 \%$ (95\% confidence interval [CI] 64\%-100\%) and its specificity, $79 \%$ (95\% CI $75 \%-83 \%)$. When these women were excluded, however, the sensitivity of CE MRI (68\%, $95 \%$ CI $45 \%-86 \%$ ) was closer to that of mammography $(50 \%$, $95 \%$ CI $28 \%-72 \% ; p=0.45)$ When results for CE MRI and

mammography were combined, the overall sensitivity was improved (Table 1), but the specificity was further reduced $(74 \%$, 95\% CI 69\%-78\%).

Commentary: This study adds to the growing evidence that CE MRI is more sensitive than mammography for detecting breast cancer in women who are genetically predisposed to the disease. It is important to develop more sensitive screening tools for this population, since the cancers that occur in these women (particularly those carrying $B R C A 1$ ) can develop rapidly, often in the intervals between normal mammographic readings at their regular screenings, and be of high grade when detected. In the present study, many cancers that were located by MRI but not by mammography had already become invasive.

There are no studies yet that demonstrate that screening with CE MRI reduces mortality from breast cancer; establishing this will take many years, as is usual with investigations of screening tests for cancer. By the time an association has been quantified, newer screening tests are likely to have been developed. Given that CE MRI appears to detect cancers that are already invasive, these are important caveats. The argument for using CE MRI to
Table 1: Performance of contrast-enhanced (CE) MRI and mammography in 3 studies involving women with a high genetic risk for breast cancer

\begin{tabular}{lcccccc}
\hline \multirow{2}{*}{$\begin{array}{l}\text { Prospective } \\
\text { cohort study }\end{array}$} & \multicolumn{2}{c}{ Sensitivity, \% (95\% confidence interval) } & & \multicolumn{2}{c}{ Specificity, \% (95\% CI) } \\
\cline { 2 - 3 } & CE MRI & Mammography & Combined & & CE MRI & Mammography \\
\hline Leach et al & $77(60-90)$ & $40(24-58)$ & $94(81-99)$ & & $81(80-83)$ & $93(92-95)$ \\
Kriege et al $^{1}$ & $71(56-81)$ & $40(26-56)$ & $89(76-96)$ & & $90(89-91)$ & $95(94-96)$ \\
Warner et al $^{2}$ & $77(55-92)$ & $36(17-59)$ & $86(65-97)$ & & $95(93-97)$ & $100(99-100)$ \\
\hline
\end{tabular}

detect breast cancer earlier is compelling, since survival rates are highest when cancers are detected in their preinvasive or early invasive stage. In the study under discussion, 29 of the 35 cancers detected were invasive. Of these 29, 20 were under $2 \mathrm{~cm}$ in diameter and at least 21 were node-negative (nodal status was unavailable in 3 cases).

Practice implications: CE MRI appears to be more sensitive than mammography for the detection of breast cancer, especially among women with the $B R C A 1$ mutation. At this time, resource limitations in Canada and elsewhere prevent its widespread use in screening. Screening with MRI is certainly much more expensive than with mammography, and not solely because the test itself costs more: the lower specificity of CE MRI would result in more women requiring further diagnostic workup after a false-positive result.

The hope for the future is that our knowledge of the patient population that will benefit most from screening with CE MRI will be improved, unnecessary patient morbidity from additional diagnostic testing reduced, cost-effectiveness realized and, most importantly, more women cured of breast cancer.

Stephen Choi

Deputy Editor, CMAf

\section{References}

1. Kriege M, Brekelmans CT, Boetes C, Besnard PE, Zonderland HM, Obdeijn IM, et al; Magnetic Resonance Imaging Screening Study Group. Efficacy of MRI and mammography for breast-cancer screening in women with a familial or genetic predisposition. N Engl 7 Med 2004;51:427-37.

2. Warner E, Plewes DB, Hill KA, Causer PA, Zubovits JT, Jong RA, et al. Surveillance of BRCA1 and BRCA2 mutation carriers with magnetic resonance imaging, ultrasound, mammography, and clinical breast examination. FAMA 2004;292(11):1317-25. 